\title{
THE FINE STRUCTURE OF TRANSITIVE RIEMANNIAN ISOMETRY GROUPS. I
}

BY

CAROLYN S. GORDON AND EDWARD N. WILSON

\begin{abstract}
Let $M$ be a connected homogeneous Riemannian manifold, $G$ the identity component of the full isometry group of $M$ and $H$ a transitive connected subgroup of $G . G=H L$, where $L$ is the isotropy group at some point of $M . M$ is naturally identified with the homogeneous space $H / H \cap L$ endowed with a suitable left-invariant Riemannian metric. This paper addresses the problem: Given a realization of $M$ as a Riemannian homogeneous space of a connected Lie group $H$, describe the structure of the full connected isometry group $G$ in terms of $H$. This problem has already been studied in case $H$ is compact, semisimple of noncompact type, or solvable. We use the fact that every Lie group is a product of subgroups of these three types in order to study the general case.
\end{abstract}

Introduction. Let $M$ be a connected homogeneous Riemannian manifold, $G$ the identity component of the full isometry group of $M$ and $H$ a transitive connected subgroup of $G$. $G=H L$, where $L$ is the (necessarily compact) isotropy group at some point of $M . M$ is naturally identified with the homogeneous space $H / H \cap L$ endowed with a suitable left-invariant Riemannian metric. We address the problems: Given a realization of $M$ as a Riemannian homogeneous space of a connected Lie group $H$, (i) describe the full connected isometry group $G$ in terms of $H$, and (ii) modify $H$ to obtain a "nicer" transitive group of isometries of $M$.

These problems have been studied in several special cases, including in particular:

(a) $H$ (and hence $M$ and $G$ ) compact [OT, On, Oz]. Oniščik gave a detailed and very complex description of decompositions of a compact Lie group $G$ into compact subgroups $G=H L$.

(b) $H$ semisimple of noncompact type [G]. In this case, $G$ is reductive and $H$ is normal in $G$.

(c) $H$ solvable [GW]. $M$ is then called a solvmanifold. $H$ can be replaced by an almost simply transitive normal subgroup $R$, which can be algorithmically modified to obtain a new almost simply transitive solvable group $S$ of isometries canonically embedded in $G$. $G$ is then described in terms of $S$.

Our goal is to piece together these special cases to study (i) and (ii) for arbitrary $H$. Our main tool is Levi's theorem which implies that every connected Lie group is a product of subgroups of the three types discussed above. Specifically, $H=H_{1} H_{2}$,

Received by the editors January 30, 1984 and, in revised form, July 20, 1984.

1980 Mathematics Subject Classification. Primary 53C 30.

Key words and phrases. Isometry groups, homogeneous Riemannian manifolds, Levi decompositions.

1985 American Mathematical Society $0002-9947 / 85 \$ 1.00+\$ .25$ per page 
where $H_{1}$ is a (nonunique) maximal semisimple subgroup, the product of maximal semisimple subgroups of noncompact and of compact type, and $\mathrm{H}_{2}$ is the solvable radical. To describe the embedding of $H$ in $G$, we proceed in two steps:

Step 1. We show that semisimple Levi factors $H_{1}$ of $H$ and $G_{1}$ of $G$ can be chosen so that $H_{1} \subset G_{1}$ and $H_{1}$ and $G_{1}$ are "compatible" with the isotropy subgroups $L_{0}=L \cap H$ of $H$ and $L$ of $G$, respectively. Here $G_{1}$ compatible with $L$ means that $G_{1} L$ is a subgroup of $G$ of the form $G_{1} L=G_{1} T$, where $T \subset G_{2}$ is abelian and commutes with $G_{1}$. We can then apply cases (a) and (b) to study the embedding of $H_{1} L_{0}$ in $G_{1} L$.

Step 2. Imitating the solvable case (c), we would ideally like to replace $\mathrm{H}_{2}$ by a solvable normal subgroup $R$ such that $H=H_{1} L_{0} R$ with $H_{1} L_{0} \cap R$ discrete. Unfortunately, such an $R$ need not exist. Instead we prove the existence of a subgroup $V$ of $H_{1} L_{0}$, central in $H$, which lies in every solvable normal subgroup $R$ of $H$ for which $H=H_{1} L_{0} R$. We then choose $R$ to have minimal intersection $V$ with $H_{1} L_{0}$ and develop a modification procedure to carry $R$ to a solvable subgroup $S$ of $G$ of the same dimension such that $H_{1} S$ is still a transitive subgroup of $G$ and $S$ is canonically embedded in $G$. In the process, we gain an understanding of the structure of $G$ and the embedding of $H$.

The present paper proves the existence in Step 1 of Levi factors compatible with the isotropy groups and examines the intersection of $H_{1} L_{0}$ with $H_{2}$, thereby establishing the existence of the central subgroup $V$ and minimal solvable group $R$ in Step 2. We also carry out a preliminary investigation of the relationship between $V$ and $R$ and the analogously defined subgroups $\tilde{V}$ and $\tilde{R}$ of groups $\tilde{H}$ lying between $H$ and $G$. The modification procedure of Step 2 will be developed in part II.

The organization of part $I$ is as follows: $\$ 1$ reviews the standard decomposition theorems of Lie groups, establishes notational conventions to be used throughout the paper, and discusses the notion of modification of solvable groups.

The existence of a semisimple Levi factor compatible with the isotropy subgroup is proved for the full connected isometry group $G$ in $\$ 2$ and for an arbitrary connected transitive group $H$ in $\S 3$. The latter case is complicated by the fact that the isotropy subgroup $L \cap H$ of $H$ need not be compact even though the full isotropy group $L$ is compact. $\S 3$ also contains a first analysis of the embedding of $H$ in $G$. In $\S 4$ we study the subgroups $R$ and $V$ of $H$ and lay the groundwork for the more intricate investigations of part II.

1. Preliminaries. We first establish the notational conventions to be used throughout the paper. A Lie group will always be denoted by a capital Roman letter and its Lie algebra by the corresponding Gothic letter.

1.1 Notation. By the Levi-Malcev theorem (see $[\mathbf{J}]$ ), every connected Lie group can be decomposed into a product of a maximal semisimple subgroup and the solvable radical. We will denote by $G_{1}$ and $G_{2}$, respectively, the semisimple Levi factor of $G$ and the solvable radical. Recall that $G_{1}$ is unique only up to conjugacy by elements of nilrad $(G)$. Given a choice of $G_{1}$, we will let $G_{\text {nc }}$ and $G_{\mathrm{c}}$ denote the maximal semisimple normal subgroups of $G$ of noncompact type and of compact type, 
respectively; i.e., $G_{\mathrm{nc}}$ and $G_{\mathrm{c}}$ are the products of all simple connected noncompact, respectively compact, normal subgroups of $G_{1}$. Thus $G=G_{1} G_{2}=G_{\mathrm{nc}} G_{\mathrm{c}} G_{2}$ and $\mathfrak{g}=\mathfrak{g}_{1}+\mathfrak{g}_{2}=\left(\mathfrak{g}_{\mathrm{nc}} \oplus \mathfrak{g}_{\mathrm{c}}\right)+\mathfrak{g}_{2}$ with $\mathfrak{g}_{1} \cap \mathfrak{g}_{2}=\{0\}$.

1.2 Reductive Lie groups. Recall that $G$ is said to be reductive if $G_{2}$ is central in $G$. In that case, $G_{1}$ is unique and normal in $G, \mathfrak{g}_{1}=[\mathfrak{g}, \mathfrak{g}]$ and $\mathfrak{g}=\mathfrak{g}_{1} \oplus \mathfrak{g}_{2}$ with $\mathfrak{g}_{2}$ equal to the center of $\mathfrak{g}$.

1.3 Compactly embedded subalgebras. A subalgebra $f$ of a Lie algebra $g$ is said to be compactly embedded in $\mathfrak{g}$ if $\mathfrak{g}$ admits an inner product relative to which the operators ad $\operatorname{ad}_{\mathfrak{g}} X, X \in \mathfrak{t}$, are skew-symmetric. If $L$ is a compact subgroup of $G$, then $\mathfrak{l}$ is compactly embedded in $\mathfrak{g}$, but the converse statement does not hold. Every compact Lie algebra is reductive. Thus $\mathfrak{f}=\mathfrak{f}_{1} \oplus \mathfrak{f}_{2}$ with $\mathfrak{f}_{1}=[\mathfrak{f}, \mathfrak{f}]$ compact and semisimple and $\mathfrak{f}_{2}$ abelian.

We next define the concept of modification, our major tool in studying solvable groups of isometries. Proofs of all assertions below may be found in [GW].

1.4 Definition. Let $r$ and $r^{\prime}$ be solvable subalgebras of a Lie algebra $g . r^{\prime}$ is said to be a modification of $r$ if there exists a linear map $\phi: r \rightarrow g$ such that $\phi(r)$ is an abelian compactly embedded subalgebra of $\mathfrak{g}, \phi(\mathfrak{r})$ normalizes $\mathfrak{r}$, and $\mathfrak{r}^{\prime}=\{X+$ $\phi(X): X \in \mathfrak{r}\} . \phi$ is then called a modification map. The modification is said to be normal if $\phi(r)$ also normalizes $r$ '.

1.5 REMARKS. The modification map associated with a given modification is not unique, but the definition of normality is independent of the choice of $\phi$. The statement that $r^{\prime}$ is a modification of $r$ is not symmetric. However, if $r^{\prime}$ is a normal modification of $\mathfrak{r}$, then $\mathfrak{r}$ is also a normal modification of $\mathfrak{r}^{\prime}$ with modification map $\psi$ given by $\psi(X+\phi(X))=-\phi(X)$ for $X \in \mathfrak{r}$.

1.6 Proposition. Let $\mathfrak{r}$ and $\mathfrak{r}^{\prime}$ be solvable subalgebras of a Lie algebra $\mathfrak{g}$, and suppose $\mathfrak{r}^{\prime}$ is a modification of $\mathfrak{r}$ with modification map $\phi$. Let $\mathfrak{n}$ denote the nilradical of r. Then:

(i) $\phi(n)$ normalizes $\mathfrak{r}^{\prime}$. In particular, if $\mathfrak{r}$ is nilpotent, every modification of $\mathfrak{r}$ is normal.

(ii) $\mathfrak{r}^{\prime}$ can be obtained from $\mathfrak{r}$ by two successive normal modifications, i.e., there exists a normal modification $\mathrm{r}^{\prime \prime}$ of $\mathrm{r}$ such that $\mathrm{r}^{\prime}$ is a normal modification of $\mathrm{r}^{\prime \prime}$.

1.7 Proposition. Suppose the connected Lie group $G$ is a product of subgroups $G=U R$ with $R$ solvable and connected. Let $\mathrm{r}^{\prime}$ be a modification of $\mathrm{r}$ in $\mathrm{g}$ and let $R^{\prime}$ be the analytic subgroup of $G$ with Lie algebra $\mathrm{r}^{\prime}$. Then $G=U R^{\prime}$.

2. Levi factors compatible with the isotropy group. Let $G$ be the identity component of the full group of isometries of a homogeneous Riemannian manifold $M$ and $L$ the isotropy subgroup at some point of $M$. $L$ is compact. However, if $H$ is a connected transitive subgroup of $G$, its isotropy subgroup $H \cap L$ need not be compact; we are only guaranteed that $\mathfrak{h} \cap \mathfrak{l}$ is compactly embedded in $\mathfrak{h}$.

2.1 Definition. Let $L$ be a subgroup of a connected Lie group $G$ such that $\mathfrak{l}$ is compactly embedded in $\mathrm{g}$. A semisimple Levi factor $G_{1}$ will be said to be compatible with $L$ if $G_{1} L$ is a reductive subgroup of $G$. Equivalently, $\mathfrak{g}_{1}+\mathfrak{l}=\mathfrak{g}_{1} \oplus \mathfrak{t}$ for some 
abelian subalgebra $t$ of $\mathfrak{g}_{2}$ commuting with $\mathfrak{g}_{1}$ (see Notation 1.1). We will also say $\mathfrak{g}_{1}$ is compatible with $\mathfrak{l}$.

In Theorem 2.6 we will prove the existence of a Levi factor $G_{1}$ of $G$ compatible with $L$ in case $L$ is actually compact. In $\S 3$ we will obtain the stronger result that if $G=H L$ with $L$ compact and $H$ connected, then $H$ contains a Levi factor $H_{1}$ compatible with $H \cap L$. In particular, any connected transitive group $H$ of isometries of a Riemannian manifold contains a semisimple Levi factor compatible with its isotropy subgroup.

2.2 LemMA. Let L be a compact subgroup of a connected Lie group $G$. There exists a semisimple Levi factor $G_{1}$ of $G$, maximal compactly embedded subalgebras $\mathfrak{f}$ of $\mathfrak{g}_{n c}$ and $\mathfrak{u}$ of $\mathfrak{g}$, and an abelian subalgebra $\mathrm{t}$ of $\mathfrak{g}_{2}$ commuting with $\mathfrak{f}+\mathfrak{g}_{\text {c }}$ such that $\mathfrak{l} \subset \mathfrak{u}=\mathfrak{f} \oplus$ $\mathfrak{g}_{c} \oplus \mathrm{t}$ ( notation as in 1.1).

Proof. Let $\operatorname{Ad}_{G}$ denote the adjoint representation of $G$ and write $\tilde{G}=\operatorname{Ad}_{G} G$. We first prove the lemma with $G$ replaced by $\tilde{G}$. Let $\tilde{G}_{1}$ be a semisimple Levi factor, $\tilde{G}_{\text {nc }}$ its noncompact part, $\tilde{f}$ a maximal compactly embedded subalgebra of $\mathfrak{g}_{n c}$, and $\tilde{K}$ the corresponding subgroup of $\tilde{G}_{\mathrm{nc}}$. The semisimple matrix group $\tilde{G}_{\mathrm{nc}}$ has inite center, so $\tilde{K}$ is compact (see [H, p. 252]). Let $\tilde{U}$ be a maximal compact subgroup of $\tilde{G}$ containing $\tilde{K} \tilde{G}_{\mathrm{c}}$. The projection $\tilde{\mathfrak{g}} \rightarrow \tilde{\mathrm{g}}$ parallel to $\tilde{\mathfrak{g}}_{2}$ sends $\tilde{\mathrm{u}}$ to a compactly embedded subalgebra of $\tilde{\mathfrak{g}}_{1}$ containing the maximal one $\tilde{\mathfrak{f}}+\tilde{\mathfrak{g}}_{c}$. It follows that $\tilde{\mathfrak{u}}=\tilde{\mathfrak{f}}+\tilde{\mathfrak{g}}_{\mathrm{c}}+\tilde{\mathfrak{t}}$ with $\tilde{\mathfrak{t}} \subset \tilde{\mathfrak{g}}_{2}$. $\tilde{\mathfrak{t}}$ is necessarily central in $\tilde{\mathfrak{u}}$ (see 1.3 ). By the conjugacy of maximal compact subgroups of $\tilde{G}$, every compact subgroup of $\tilde{G}$ satisfies the conclusion of the lemma.

$\operatorname{Ad}_{G}(L)$ lies in some maximal compact subgroup $\tilde{U}$ of $\tilde{G}$ with $\tilde{\mathfrak{u}}=\tilde{\mathfrak{f}} \oplus \tilde{\mathfrak{g}}_{\mathrm{c}} \oplus \tilde{\mathrm{f}}$ as above. Let $\mathfrak{u}=\left(\operatorname{ad}_{\mathfrak{H}}\right)^{-1} \tilde{\mathfrak{u}}$. $\mathfrak{u}$ is compactly embedded in $\mathfrak{g}$ since $\tilde{U}=\operatorname{Ad}_{G}(U)$ is compact. $\tilde{\mathfrak{g}}_{1}=\operatorname{ad}_{\mathfrak{g}} \mathfrak{g}_{1} \simeq \mathfrak{g}_{1}$ for some semisimple Levi factor $\mathfrak{g}_{1}$, so $\mathfrak{u}=\mathfrak{f}+\mathfrak{g}_{\mathrm{c}}+\mathfrak{t}$ with $\mathfrak{f}$ a maximal compact subalgebra of $\mathfrak{g}_{n c}$ and $t=\left(\operatorname{ad}_{\mathfrak{q}}\right)^{-1} \mathfrak{f}=\mathfrak{u} \cap \mathfrak{g}_{2}$. Again, by $1.3, \mathrm{t}$ is central in $u$.

Before proving the existence of Levi factors compatible with $L$, we establish some elementary properties which will be needed later. Proposition 2.3 can be trivially checked in the case of the specific compatible Levi factor which will be constructed in 2.6 .

2.3 Proposition. Let $\mathfrak{l}$ be a compactly embedded subalgebra of $\mathrm{g}$ and let $\mathrm{g}_{1}$ be a Levi factor of $\mathrm{g}$ compatible with $\mathfrak{l}$. Let $\mathrm{t}=\left(\mathfrak{g}_{1}+\mathfrak{l}\right) \cap \mathfrak{g}_{2}$ and let $\pi_{1}: \mathfrak{g} \rightarrow \mathfrak{g}_{1}$ be the homomorphic projection. Then $\pi_{1}(\mathfrak{l})+\mathrm{t}$ is compactly embedded in $\mathfrak{g}$.

Proof. $\pi_{1}(\mathfrak{l})$ is compactly embedded in $\mathfrak{g}_{1}$, and hence lies in $\mathfrak{f}+\mathfrak{g}_{\mathrm{c}}$ for some maximal compactly embedded subalgebra ${ }^{*}$ of $g_{1}$. Let $G$ be a connected Lie group with Lie algebra g. Then $\operatorname{Ad}_{G}\left(K G_{\mathrm{c}}\right.$ ) is compact (see the proof of 2.2). Let $\langle, \quad>$ be an inner product on $\mathfrak{g}$ relative to which the operators $\operatorname{ad}_{\mathfrak{a}} X, X \in \mathfrak{l}_{2}$, are skew-symmetric. Let $\mathfrak{b}=\pi_{1}(\mathfrak{l}) \supset \mathfrak{l}_{1}, B$ the corresponding subgroup of $K G_{\mathfrak{c}}$, and $C$ the closure of $\operatorname{Ad}_{G}(B)$ in $\operatorname{Ad}_{G}\left(K G_{\mathrm{c}}\right)$. Since $\mathfrak{g}_{1}+\mathfrak{l}$ is reductive,

$$
\left[\pi_{1}(\mathfrak{l}), \mathfrak{l}_{2}\right]=\left[\pi_{1}(\mathfrak{l}), \pi_{1}\left(\mathfrak{l}_{2}\right)\right]=\pi_{1}\left[\mathfrak{l}, \mathfrak{l}_{2}\right]=\{0\}
$$


so $C$ commutes with $\operatorname{ad}_{\mathfrak{g}} \mathfrak{l}_{2}$. Hence both $\pi_{1}(\mathfrak{l})$ and $\mathfrak{l}_{2}$, and consequently $\mathfrak{t}$, are skew-symmetric relative to the inner product on $g$ obtained by averaging $\langle$, over $C$.

2.4 Proposition. Let $\mathfrak{l}$ be a compactly embedded subalgebra of $\mathfrak{g}$ and let $z(\mathrm{~g})$ denote the center of $\mathfrak{g}$. If $\mathfrak{g}_{1}$ and $\mathrm{g}_{1}^{\prime}$ are semisimple Levi factors compatible with $\mathfrak{l}$, then

$$
\left(\mathfrak{g}_{1}+\mathfrak{l}\right) \cap z(\mathfrak{g})=\left(\mathfrak{g}_{1}^{\prime}+\mathfrak{l}\right) \cap z(\mathfrak{g}) .
$$

2.5 REMARK. Suppose $G$ is a transitive group of isometries of a Riemannian manifold $M$ and $L$ is its isotropy subgroup. Let $Y \in\left(g_{1}+\mathfrak{l}\right) \cap z(\mathfrak{g})$. Then $X+Y$ $\in \mathfrak{l}_{2}$ for some $X \in \mathfrak{g}_{1}, \operatorname{ad}_{\mathfrak{g}} X=\operatorname{ad}_{\mathfrak{g}}(X+Y)$, and $\operatorname{ad}_{\mathfrak{g}} X$ commutes with the isotropy action of $\mathfrak{l}$. For $B$ the one-parameter subgroup of $G$ generated by $X, \operatorname{Ad}_{G} B$ induces a well-defined isometric action of $B$ on $M=G / L$.

Proof of Proposition 2.4. Let

$$
\mathfrak{t}=\left(\mathfrak{g}_{1}+\mathfrak{l}\right) \cap \mathfrak{g}_{2}, \quad \mathfrak{t}^{\prime}=\left(\mathfrak{g}_{1}^{\prime}+\mathfrak{l}\right) \cap \mathfrak{g}_{2}
$$

and

$$
\mathfrak{w}=\left(\mathfrak{g}_{1}+\mathfrak{l}\right) \cap z(\mathfrak{g})=\mathfrak{t} \cap z(\mathfrak{g}), \quad \mathfrak{w}^{\prime}=\left(\mathfrak{g}_{1}^{\prime}+\mathfrak{l}\right) \cap z(\mathfrak{g})=\mathfrak{t}^{\prime} \cap z(\mathfrak{g}) .
$$

Let $\pi_{1}: \mathfrak{g} \rightarrow \mathrm{g}_{1}$ and $\pi_{1}^{\prime}: \mathfrak{g} \rightarrow \mathfrak{g}_{1}^{\prime}$ be the homomorphic projections parallel to $\mathfrak{g}_{2}$. Then

$$
\mathfrak{t}=\left\{Y-\pi_{1}(Y): Y \in \mathfrak{l}\right\}, \quad \mathfrak{t}^{\prime}=\left\{Y-\pi_{1}^{\prime}(Y): Y \in \mathfrak{l}\right\} .
$$

Let $\mathfrak{n}=\operatorname{nilrad}(g)$. By the Levi-Malcev theorem, there exists $U \in \mathfrak{n}$ such that $g_{1}=\alpha\left(g_{1}^{\prime}\right)$ for $\alpha=e^{\text {ad } U}$. Fix $W \in \mathfrak{w}$. By (2.3), $W=Y-X$ with $Y \in \mathfrak{l}$ and $X=$ $\pi_{1}(Y)$. Since $\mathfrak{g}_{1} \subset \mathfrak{g}_{1}^{\prime}+\mathfrak{n}, X=X_{1}^{\prime}+X_{2}^{\prime}$ with $X_{1}^{\prime} \in \mathfrak{g}_{1}^{\prime}, X_{2}^{\prime} \in \mathfrak{n}$. Since $W \in z(\mathfrak{g}) \subset$ $\mathfrak{g}_{2}$,

$$
0=\pi_{1}^{\prime}(W)=\pi_{1}^{\prime}(Y)-X_{1}^{\prime}
$$

so $X_{1}^{\prime}=\pi_{1}^{\prime}(Y) \in \pi_{1}^{\prime}(\mathfrak{l})$. Moreover, since $X_{2}^{\prime}=Y-X_{1}^{\prime}-W$, ad $X_{2}^{\prime}=\operatorname{ad}\left(Y-X_{1}^{\prime}\right)$ is both nilpotent $\left(X_{2}^{\prime} \in \mathfrak{n}\right)$ and skew-symmetric $\left(Y-X_{1}^{\prime} \in \mathfrak{l}+\pi_{1}^{\prime}(\mathfrak{l})=\pi_{1}^{\prime}(\mathfrak{l})+\mathfrak{t}^{\prime}\right.$, a compactly embedded subalgebra by Proposition 2.3). Hence ad $X_{2}^{\prime}=0$, i.e., $X_{2}^{\prime} \in$ $z(\mathrm{~g})$ and $\alpha\left(X_{2}^{\prime}\right)=X_{2}$. Since $W \in z(\mathrm{~g})$,

$$
Y-X=W=\alpha(W)=\alpha(Y)-\alpha\left(X_{1}^{\prime}\right)-X_{2}^{\prime},
$$

so

$$
\alpha\left(X_{1}^{\prime}\right)-X=\alpha(Y)-Y-X_{2}^{\prime} .
$$

The left-hand side of (2.4) lies in $\mathfrak{g}_{1}$ and the right-hand side lies in $\mathfrak{n} . n \cap \mathfrak{g}_{1}=\{0\}$, so $X=\alpha\left(X_{1}^{\prime}\right)$ and

$$
X_{2}^{\prime}=X-X_{1}^{\prime}=e^{\operatorname{ad} U}\left(X_{1}^{\prime}\right)-X_{1}^{\prime}=\left[U, X_{1}^{\prime}\right]+\sum_{k \geqslant 2} \frac{(\operatorname{ad} U)^{k}}{k !} X_{1}^{\prime} .
$$

Let $\mathfrak{n}_{j}$ be the $j$ th ideal in the lower central series of $n$ and choose an $\operatorname{ad}\left(g_{1}^{\prime}\right)$-invariant subspace $\mathfrak{m}_{j}$ of $\mathfrak{n}_{j}$ such that $\mathfrak{n}_{j}=\mathfrak{m}_{j}+\mathfrak{n}_{j+1}$ (vector space direct sum). Write $U=\sum_{j \geqslant 1} U_{j}$ with $U_{j} \in \mathrm{m}_{j}$. If $\left[U, X_{1}^{\prime}\right] \neq 0$, let $j_{0}$ be the first index for which $\left[U_{j_{0}}, X_{1}^{\prime}\right] \neq 0$. Then $X_{2}^{\prime} \equiv\left[U_{j_{0}}, X_{1}^{\prime}\right] \bmod \mathfrak{n}_{j+1}$ and

$$
0=\left[X_{1}^{\prime}, X_{2}^{\prime}\right] \equiv-\left(\operatorname{ad} X_{1}^{\prime}\right)^{2}\left(U_{j_{0}}\right) \bmod \mathfrak{n}_{j+1} \text {. }
$$


Since $m_{j}$ is $\operatorname{ad}\left(\mathfrak{g}_{1}^{\prime}\right)$-invariant, it follows that $\left(\operatorname{ad} X_{1}^{\prime}\right)^{2}\left(U_{j_{0}}\right)=0$. By semisimplicity of ad $X_{1}^{\prime}\left(X_{1}^{\prime}\right.$ lies in the compactly embedded algebra $\left.\pi_{1}^{\prime}(\mathfrak{l})\right),\left[X_{1}^{\prime}, U_{j_{0}}\right]=0$, contradicting the definition of $j_{0}$. Thus $\left[U, X_{1}^{\prime}\right]=0$ and $X_{2}^{\prime}=0$ by (2.5), i.e., $X=X_{1}^{\prime}$ and $W=Y-X_{1}^{\prime} \in \mathfrak{g}_{1}^{\prime}+\mathfrak{l}$. Hence $\mathfrak{w} \subset \mathfrak{w}^{\prime}$ and equality holds by symmetry.

2.6 THEOREM. If $L$ is a compact subgroup of a connected Lie group $G$, then $G$ contains a semisimple Levi factor $G_{1}$ compatible with $L$.

Proof. Choose $\mathfrak{g}_{1}, \mathfrak{f}, \mathfrak{t}$ as in Lemma 2.2 so that $\mathfrak{l} \subset \mathfrak{u}=\mathfrak{f} \oplus \mathfrak{g}_{\mathfrak{c}} \oplus \mathfrak{t}$. Note that $\mathfrak{l}_{1}=[\mathfrak{l}, \mathfrak{l}] \subset[\mathfrak{u}, \mathfrak{u}] \subset \mathfrak{g}_{1}$. Shrink $\mathfrak{t}$ if necessary so that $\mathfrak{u}=\mathfrak{l}+\mathfrak{g}_{\mathfrak{c}}+\mathfrak{l}$. $\mathfrak{t}$ need not centralize all of $\mathrm{g}$. We will need to make a series of replacements in order to obtain a new Levi factor which commutes with $t$ and still contains $\mathfrak{f}+\mathfrak{g}_{\mathrm{c}}$.

Fix an inner product on $\mathfrak{g}$ relative to which the operators $\operatorname{ad}_{\mathfrak{g}} X, X \in \mathfrak{u}$, are skew-symmetric. Then $\delta=\left\{\operatorname{ad}_{\mathfrak{g}} X: X \in \mathrm{t}\right\}$ is an abelian Lie algebra of skew-symmetric derivations of $\mathfrak{g}$. Fix $D \in \mathfrak{d}$ for which the abelian associative algebra generated by $D$ coincides with that generated by $\mathfrak{d}$, e.g., choose $D \in \mathfrak{d}$ for which $\mu(D) \neq \nu(D)$ for all weights $\mu, \nu \in \mathfrak{d}^{\prime}$ of the action of $\delta$ on $\mathfrak{g} . D\left(\mathfrak{g}_{1}\right) \subset\left[\mathfrak{g}_{1}, \mathfrak{g}_{2}\right] \subset \mathfrak{n}$, where $\mathfrak{n}$ denotes the nilradical of $\mathrm{g}$. Let $\left(n_{j}\right)_{j \geqslant 1}$ be the lower central series of $\mathfrak{n}$. Since the action of $\operatorname{ad}_{\mathfrak{a}} \mathfrak{g}_{1}$ is completely reducible, we may choose for each $j$ a subspace $m_{j} \subset n_{j}$ such that $n_{j}=m_{j}+n_{j+1}$ (vector space direct sum) and $\left[g_{1}, m_{j}\right]$ $\subset \mathfrak{m}_{j} . D\left(\mathfrak{g}_{1}\right) \subset \mathfrak{n}_{j}$ for some $j$ and we may write for $X \in \mathfrak{g}_{1}, D(X)=f(X)+g(X)$ with $f, g$ linear maps from $\mathfrak{g}_{1}$ into $\mathfrak{m}_{j}$ and $\mathfrak{n}_{j+1}$, respectively. Since $D$ is a derivation of $\mathfrak{g}$ and $\left[\mathfrak{g}, \mathfrak{n}_{j+1}\right] \subset \mathrm{n}_{j+1}$,

$$
f[X, Y]-[f(X), Y]-[X, f(Y)]=0,
$$

i.e., $f$ defines an element in the relative Lie algebra cohomology group $H^{1}\left(\mathfrak{g}_{1} ; \mathfrak{m}_{j}\right)$. The semisimplicity of $g_{1}$ implies that all such cohomology groups are trivial and thus there exists $U \in \mathfrak{m}_{j}$ such that $f(X)=[U, X]$ for all $X \in \mathfrak{g}_{1}$.

Let $\mathfrak{v}$ be the smallest $\operatorname{ad}\left(\mathfrak{g}_{1}\right)$-invariant subspace of $\mathfrak{m}_{j}$ containing $\left[U, \mathfrak{g}_{1}\right] . \mathfrak{v}=$ $\sum_{k=1}^{\infty} \mathfrak{v}_{k}$ with $\mathfrak{v}_{1}=\left[U, \mathfrak{g}_{1}\right]$ and $\mathfrak{v}_{k+1}=\left[\mathfrak{v}_{k}, \mathfrak{g}_{1}\right]$ for $k \geqslant 1$. Both $\mathfrak{v}$ and $R U+\mathfrak{v}$ are $\operatorname{ad}\left(\mathfrak{g}_{1}\right)$-invariant, so by semisimplicity of $\mathfrak{g}_{1}, U=U^{\prime}+U^{\prime \prime}$ with ad $\mathfrak{g}_{1}\left(U^{\prime}\right)=0$ and $U^{\prime \prime} \in \mathfrak{v}$. Replacing $U$ by $U^{\prime \prime}$, we may assume $U \in \mathfrak{v}$.

We claim that $\mathfrak{v} \subset D m_{j}+n_{j+1}$. Let $g_{2}^{\frac{1}{2}}$ be the orthogonal complement of $\mathfrak{g}_{2}$ in $\mathfrak{g}$ relative to the inner product chosen above. Then there exists a linear map $\phi$ : $\mathfrak{g}_{1} \rightarrow \mathfrak{g}_{2}$ such that $\mathfrak{g}_{2}^{\perp}=\left\{X-\phi(X): X \in \mathfrak{g}_{1}\right\}$. Since $D(\mathfrak{g}) \subset \mathfrak{g}_{2}$ and $D$ is skew-symmetric, $D$ vanishes on $\mathfrak{g}_{2} \frac{1}{2}$ and thus $[U, X]=D(\phi(X))-g(X)$ for all $X \in \mathfrak{g}_{1}$. Hence $\mathfrak{v}_{1} \subset D \mathfrak{g}_{2}+\mathfrak{n}_{j+1}$. Being a derivation, $D$ normalizes both $\mathfrak{n}_{j}$ and $\mathfrak{n}_{j+1}$. Since $\mathfrak{v}_{1} \subset \mathfrak{n}_{j}$, we conclude that $\mathfrak{b}_{1} \subset D \mathfrak{m}_{j}+\mathfrak{n}_{j+1}$. Now assume inductively that $\mathfrak{b}_{k} \subset D \mathfrak{m}_{j}+\mathfrak{n}_{j+1}$. Then

$$
\mathfrak{v}_{k+1}=\left[\mathfrak{v}_{k}, \mathfrak{g}_{1}\right] \subset\left[D \mathfrak{m}_{j}+\mathfrak{n}_{j+1}, \mathfrak{g}_{1}\right] \subset\left[D \mathfrak{m}_{j}, \mathfrak{g}_{1}\right]+\mathfrak{n}_{j+1} .
$$

But $\left[D \mathfrak{m}_{j}, \mathfrak{g}_{1}\right] \subset D\left[\mathfrak{m}_{j}, \mathfrak{g}_{1}\right]+\left[\mathfrak{m}_{j}, D \mathrm{~g}_{1}\right] \subset D \mathfrak{m}_{j}+\left[\mathfrak{m}_{j}, \mathfrak{n}_{j}\right] \subset D \mathfrak{m}_{j}+\mathfrak{n}_{j+1}$. Thus $\mathfrak{v}_{k+1} \subset D m_{j}+n_{j+1}$ and the claim follows by induction.

Since $U \in \mathfrak{v} \subset D \mathfrak{m}_{j}+\mathfrak{n}_{j+1}$, there exists $V \in \mathfrak{m}_{j}$ such that $U-D V \in \mathfrak{n}_{j+1} . D$ vanishes on $\mathfrak{f}+\mathfrak{g}_{\mathfrak{c}}$, so $\left[U, \mathfrak{f}+\mathfrak{g}_{\mathrm{c}}\right]=\{0\}$. Also $D_{\mid \mathfrak{g}_{2}}$ commutes with $\operatorname{ad}\left(\mathfrak{f}+\mathfrak{g}_{\mathrm{c}}\right)_{\mid \mathfrak{g}_{2}}$. Let $\mathfrak{v}$ be the 0 -eigenspace of $\operatorname{ad}\left(\mathfrak{f}+\mathfrak{g}_{\mathfrak{c}}\right)$ in $\mathfrak{m}_{j}$ and let $\mathfrak{w}=\left[\mathfrak{f}+\mathfrak{g}_{c}, \mathfrak{m}_{j}\right]$. Modulo 
$\mathfrak{n}_{j+1}, D$ leaves both $\mathfrak{o}$ and $\mathfrak{m}$ invariant. Note that $U \in \mathfrak{o}$. Write $V=V^{\prime}+V^{\prime \prime}$ with $V^{\prime} \in \mathfrak{o}, V^{\prime \prime} \in \mathfrak{w}$. Modulo $\mathfrak{n}_{j+1}, U \equiv D V^{\prime}+D V^{\prime \prime}$ with $D V^{\prime} \in \mathfrak{o}, D V^{\prime \prime} \in \mathfrak{w}$. Thus $D V^{\prime \prime} \equiv 0 \bmod \mathfrak{n}_{j+1}$ and we may replace $V$ by $V^{\prime}$. Thus we assume $\left[\mathfrak{f}+\mathfrak{g}_{c}, V\right]=\{0\}$. For $X \in \mathfrak{g}_{1}$, modulo $\mathfrak{n}_{j+1}$,

$$
\begin{aligned}
D\left(e^{-\operatorname{ad} v} X\right) & \equiv D(X-[V, X]) \equiv[U, X]-[D V, X]-[V, D X] \\
& \equiv[U-D V, X] \equiv 0 .
\end{aligned}
$$

Therefore $\mathrm{g}_{1}^{\prime}=e^{-\mathrm{ad} V}\left(\mathrm{~g}_{1}\right)$ is a Levi factor of $\mathrm{g}$ with $D\left(\mathfrak{g}_{1}^{\prime}\right) \subset \mathfrak{n}_{j+1}$. Moreover, since $V \in \mathfrak{o}, \mathfrak{g}_{1}^{\prime}$ contains $f+\mathfrak{g}_{\mathrm{c}}$. By induction on $j$, there is an inner automorphism $\gamma$ of $\mathrm{g}$ such that $\gamma\left(g_{1}\right)$ is a Levi factor containing $+g_{c}$ and $D\left(\gamma\left(g_{1}\right)\right)=\{0\}$. Recall that $\mathfrak{l} \subset \mathfrak{f}+\mathfrak{g}_{\mathrm{c}}+\mathfrak{t}$ with $\mathfrak{t}$ an abelian subalgebra of $\mathfrak{g}_{2}$. By our choice of $D,\left[\mathfrak{t}, \gamma\left(\mathfrak{g}_{1}\right)\right]=$ $\{0\}$. Thus $\gamma\left(g_{1}\right)+\mathfrak{l}=\gamma\left(g_{1}\right)+t$ is a reductive subalgebra of $g$.

2.7 Remark. If $H$ is a connected subgroup of $G$ satisfying $G=H L$, we may choose the Levi factor $G_{1}$ compatible with $L$ so that $G_{1}$ also contains a Levi factor $H_{1}$ of $H$. (However, $H_{1}$ is not a priori compatible with $H \cap L$.) Indeed, let $H_{1}^{\prime}$ be any Levi factor of $H$ and choose a Levi factor $G_{1}^{\prime}$ containing $H_{1}^{\prime}$. By the conjugacy of Levi factors, there exists $x \in G$ such that $x G_{1}^{\prime} x^{-1}$ is compatible with $L . x=x^{\prime} x^{\prime \prime}$ with $x^{\prime} \in L, x^{\prime \prime} \in H .\left(x^{\prime}\right)^{-1}\left(x G_{1}^{\prime} x^{-1}\right) x^{\prime}=x^{\prime \prime} G_{1}^{\prime}\left(x^{\prime \prime}\right)^{-1}$ is also compatible with $L$ and contains the Levi factor $x^{\prime \prime} H_{1}^{\prime}\left(x^{\prime \prime}\right)^{-1}$ of $H$.

3. Embedding of $H_{\mathrm{nc}}$. We can now give a detailed description of the embedding of $H_{\mathrm{nc}}$ in $G$ and a preliminary description of the embeddings of $H_{\mathrm{c}}$ and $H_{2}$ in $G$ for some Levi decomposition $H=\left(H_{\mathrm{nc}} H_{\mathrm{c}}\right) H_{2}$. We will then use this description to prove the existence of a semisimple Levi factor of $H$ compatible with $H \cap L$. See [H] for a discussion of Iwasawa decompositions.

3.1 TheOREM. Let $G$ be a connected Lie group containing subgroups $H$ and $L$ such that $G=H L$ and $L$ is compact. Choose Levi factors $\mathfrak{h}_{1}$ of $\mathfrak{h}$ and $\mathfrak{g}_{1}$ of $g$ such that $\mathfrak{h}_{1} \subset \mathfrak{g}_{1}$, and $\mathfrak{g}_{1}$ is compatible with $\mathfrak{l}\left(\right.$ see 2.7). Let $\mathfrak{g}_{n c}$ and $\mathfrak{g}_{c}$ denote the noncompact and compact parts of $\mathrm{g}_{1}$. Then for a suitably chosen Iwasawa decomposition $\mathfrak{g}_{n c}=\mathfrak{f}+$ $\mathfrak{a}+\mathfrak{n}$, we have the following:

(i) $\mathfrak{h}_{n c} \subset \mathfrak{g}_{n c} \cdot \mathfrak{h}_{n c}$ has Iwasawa decomposition $\mathfrak{h}_{n c}=\mathfrak{l}^{\prime}+\mathfrak{a}^{\prime}+\mathfrak{n}^{\prime}$ with $\mathfrak{f}^{\prime} \subset \mathfrak{f}$, $a^{\prime} \subset \mathfrak{a}, \mathfrak{n}^{\prime} \subset \mathfrak{n}$.

(ii) There exists ideals $\mathfrak{a}^{\prime \prime}$ of $\mathfrak{a}$ and $\mathfrak{n}^{\prime \prime}$ of $\mathfrak{n}$ such that $\mathfrak{a}=\mathfrak{a}^{\prime}+\mathfrak{a}^{\prime \prime}$ and $\mathfrak{n}=\mathfrak{n}^{\prime}+\mathfrak{n}^{\prime \prime}$ (vector space direct sums). $\left[\mathfrak{a}^{\prime \prime}, \mathfrak{n}^{\prime \prime}\right]=\mathfrak{n}^{\prime \prime}$ and $\mathfrak{a}^{\prime \prime}+\mathfrak{n}^{\prime \prime}$ is an ideal of $\mathfrak{a}+\mathfrak{n}$. Let $m$ denote the centralizer of $\mathfrak{a}$ in $\mathfrak{f}$ (equivalently, the normalizer of $\mathfrak{a}+\mathfrak{n}$ in $\mathfrak{f}$ ). $m$ normalizes $\mathfrak{a}^{\prime \prime}+\mathfrak{n}^{\prime \prime}$ and splits into a direct sum of ideals $\mathfrak{m}=\mathfrak{m}^{\prime} \oplus \mathfrak{m}^{\prime \prime}$ with $\mathfrak{m}^{\prime} \subset \mathfrak{h}_{n c}$ and $\left[\mathfrak{h}_{n c}, \mathfrak{m}^{\prime \prime}\right]=\{0\}$.

(iii) For $\pi_{n c}: \mathfrak{g} \rightarrow \mathfrak{g}_{n c}$ the projection parallel to $\mathfrak{g}_{c}+\mathfrak{g}_{2}, \pi_{n c}\left(\mathfrak{h}_{2}\right)$ contains a normal modification by elements of $\mathfrak{m}^{\prime \prime}$ of $\mathfrak{a}^{\prime \prime}+\mathfrak{n}^{\prime \prime}\left(\right.$ see 1.4). In particular, $\mathfrak{n}^{\prime \prime} \subset \pi_{n c}\left(\mathfrak{h}_{2}\right)$.

(iv) $\pi_{n c}\left(\mathfrak{h}_{c}\right) \subset \mathfrak{m}^{\prime \prime}$. Thus $\pi_{n c}(\mathfrak{h}) \subset \mathfrak{h}_{n c}+\mathfrak{m}^{\prime \prime}+\mathfrak{a}^{\prime \prime}+\mathfrak{n}^{\prime \prime}=\mathfrak{h}_{n c}+\mathfrak{m}+\mathfrak{a}+\mathfrak{n}$.

(v) Let $\Delta$ be the roots of $a$ in $\mathfrak{g}_{n c}$ and $\pi$ a base for the positive roots $\Delta^{+}$. For $\alpha \in \Delta$, let $\mathfrak{g}_{\alpha}$ denote the associated root space. There exists a subset $\pi^{\prime}$ of $\pi$ such that $\mathfrak{h}_{n c}$ is generated by $\sum_{ \pm \alpha \in \pi^{\prime}} \mathrm{g}_{\alpha}$. 
(vi) Let $\Delta^{\prime}$ be the subset of $\Delta$ spanned by $\pi^{\prime}$ and let $\Delta^{\prime \prime}$ be the complement of $\Delta^{\prime}$ in $\Delta$. Let $\theta$ be the Cartan involution of $\mathfrak{g}_{n}$ fixing $\mathfrak{f}$. Then

$$
\begin{aligned}
& \mathfrak{a}^{\prime}=\sum_{\alpha \in \pi^{\prime}}\left[\mathfrak{g}_{\alpha}, \theta \mathfrak{g}_{\alpha}\right], \quad \mathfrak{n}^{\prime}=\sum_{\alpha \in\left(د^{\prime}\right)^{+}} \mathfrak{g}_{\alpha}, \\
& \mathfrak{a}^{\prime \prime}=\left\{H \in \mathfrak{a}: \alpha(H)=0 \text { for all } \alpha \in \pi^{\prime}\right\}, \quad \mathfrak{n}^{\prime \prime}=\sum_{\alpha \in\left(\Delta^{\prime \prime}\right)^{+}} \mathfrak{g}_{\alpha} .
\end{aligned}
$$

3.2 LEMMA. If $\mathfrak{h}$ is a subalgebra of $\mathfrak{g}$, and $\mathfrak{h}_{1}$ and $\mathfrak{g}_{1}$ are Levi factors of $\mathfrak{h}$ and $\mathfrak{g}$ with $\mathfrak{h}_{1} \subset \mathfrak{g}_{1}$, then $\mathfrak{h}_{n c} \subset \mathfrak{g}_{n c}$.

Proof. Let $\pi_{\mathrm{c}}: \mathfrak{g} \rightarrow \mathfrak{g}_{\mathrm{c}}$ be the homomorphic projection parallel to $\mathfrak{g}_{\mathrm{nc}}$. Since the compact algebra $\mathfrak{g}_{c}$ contains no noncompact semisimple subalgebras, $\pi_{c}\left(\mathfrak{h}_{n c}\right)=\{0\}$ and $\mathfrak{h}_{\mathrm{nc}} \subset \mathfrak{g}_{\mathrm{nc}}$.

Proof of Theorem 3.1. Let $\tilde{\pi}_{\mathrm{nc}}: G \rightarrow G /\left(G_{\mathrm{c}} G_{2} D\right)$ be the projection where $D$ is the discrete center of $G_{\mathrm{nc}}$. Under identification of the Lie algebra of $\tilde{\pi}_{\mathrm{nc}}(G)$ with $\mathrm{g}_{\mathrm{nc}}, \tilde{\pi}_{\mathrm{nc}}$ has differential $\pi_{\mathrm{nc}} \cdot \pi_{\mathrm{nc}}\left(\mathfrak{h}_{\mathrm{nc}}\right)=\mathfrak{h}_{\mathrm{nc}}$ by Lemma 3.2. $\tilde{\pi}_{\mathrm{nc}}(G)=\tilde{\pi}_{\mathrm{nc}}(L) \tilde{\pi}_{\mathrm{nc}}(H)$. $\tilde{\pi}_{\mathrm{nc}}(L)$ lies in a maximal compact subgroup $\tilde{K}$ of $\tilde{\pi}_{\mathrm{nc}}(G) . \tilde{\pi}_{\mathrm{nc}}(G) / \tilde{K}$ with any left-invariant Riemannian metric is a symmetric space of noncompact type on which $\tilde{\pi}_{\mathrm{nc}}(H)$ acts transitively by isometries.

In [GW] we classified all transitive groups of isometries of symmetric spaces of noncompact type as follows: Let $G\left(=G_{\mathrm{nc}}\right)$ be the full isometry group of such a symmetric space and $G=K A N$ an Iwasawa decomposition. For $\Delta, \pi$ and $\mathfrak{g}_{\alpha}$ as in (v) and for $\pi^{\prime}$ any subset of $\pi, \sum_{ \pm \alpha \in \pi^{\prime}} \mathfrak{g}_{\alpha}$ generates a semisimple Lie algebra $\mathfrak{b}\left(\pi^{\prime}\right)$ of noncompact type. The centralizer $m$ of $a$ in $f$ and the algebras $a^{\prime}, a^{\prime \prime}, \mathfrak{n}^{\prime}$ and $\mathfrak{n}^{\prime \prime}$ associated to $\pi^{\prime}$ as in (vi) satisfy the conclusions of (ii). A subalgebra $u$ of $g$ is the Lie algebra of a transitive subgroup of $G$ if and only if there exists an Iwasawa decomposition of $G$ and a set $\pi^{\prime}$ as above such that

$$
\mathfrak{b}\left(\pi^{\prime}\right)+\mathfrak{r}^{\prime \prime} \subset \mathfrak{u} \subset \mathfrak{b}\left(\pi^{\prime}\right)+\mathfrak{m}^{\prime \prime}+\mathfrak{a}^{\prime \prime}+\mathfrak{n}^{\prime \prime}
$$

for some normal modification $r^{\prime \prime}$ of $a^{\prime \prime}+n^{\prime \prime}$ by elements of $m^{\prime \prime}$. When (3.1) holds, $\mathfrak{u}_{n c}=\mathfrak{b}\left(\pi^{\prime}\right), \mathfrak{u}_{2}$ contains a normal modification of $a^{\prime \prime}+\mathfrak{n}^{\prime \prime}$ and $\mathfrak{u}_{c} \subset\left[\mathfrak{m}^{\prime \prime}, \mathfrak{m}^{\prime \prime}\right]$. Theorem 3.1 follows.

3.3 THEOREM. Suppose that $H$ is a connected subgroup and L a compact subgroup of a connected Lie group $G$ and that $G=H L$. Then there exists semisimple Levi factors $H_{1} \subset H$ and $G_{1} \subset G$ such that $H_{1}$ is compatible with $H \cap L$ and $G_{1}$ is compatible with $L$.

Proof. It suffices to prove the theorem with $L$ replaced by any conjugate $x L x^{-1}$, $x \in G$. Indeed, writing $x=x^{\prime} x^{\prime \prime}$ with $x^{\prime} \in H, x^{\prime \prime} \in L$, we then obtain Levi factors $\left(x^{\prime}\right)^{-1} H_{1} x^{\prime}$ and $\left(x^{\prime}\right)^{-1} G_{1} x^{\prime}$ compatible with $L \cap H$ and $L$, respectively.

We use the notation of Theorem 3.1. Thus $\mathfrak{g}_{1}$ is a Levi factor of $g$ compatible with $\mathfrak{l}, \mathfrak{g}_{\mathrm{nc}}$ has Iwasawa decomposition $\mathfrak{f}+\mathfrak{a}+\mathfrak{n}, \mathfrak{h}_{1}$ is a Levi factor of $\mathfrak{h}$ contained in $\mathfrak{g}_{1}$ and $\mathfrak{h}_{n c}=\mathfrak{l}^{\prime}+\mathfrak{a}^{\prime}+\mathfrak{n}^{\prime}$ as in 3.1(i). $\mathfrak{g}_{1}+\mathfrak{l}=\mathfrak{g}_{1} \oplus \mathfrak{t}$ with $\mathrm{t}$ abelian, and $\mathfrak{l} \subset \tilde{f}+\mathfrak{g}_{\mathfrak{c}}$ $+\mathrm{t}$, where $\tilde{\mathrm{f}}$ is a maximal compact subalgebra of $\mathrm{g}_{\mathrm{nc}}$. We may choose $x \in G_{\mathrm{nc}}$ such that $\mathfrak{f}=\operatorname{Ad}(x) \tilde{\mathfrak{f}}$. Since $\left[\mathfrak{g}_{\mathrm{nc}}, \mathfrak{g}_{\mathrm{c}}+\mathrm{t}\right]=\{0\}, \operatorname{Ad}(x) \mathfrak{l} \subset \mathfrak{f}+\mathfrak{g}_{\mathrm{c}}+\mathfrak{t}$. As noted above, 
we may replace $\mathfrak{l}$ with $\operatorname{Ad}(x) \mathfrak{l}$. Let $\mathfrak{u}=\mathfrak{H}+\mathfrak{g}_{\mathfrak{c}}+\mathfrak{t}$ and $\mathfrak{u}^{\prime}=\mathfrak{u} \cap \mathfrak{h}$. $\mathfrak{u}^{\prime}$ is a compactly embedded subalgebra of $\mathfrak{h}$ containing both $\mathfrak{g}_{\mathfrak{c}}$ and $\mathfrak{l} \cap \mathfrak{h}$. By Theorem 3.1,

$$
\mathfrak{u}^{\prime}=\mathfrak{l}^{\prime}+\left\{\left(\mathfrak{m}^{\prime \prime}+\mathfrak{g}_{\mathfrak{c}}+\mathfrak{t}\right) \cap \mathfrak{h}\right\}
$$

and

$$
\begin{gathered}
{\left[\mathfrak{u}^{\prime}, \mathfrak{h}_{n c}\right] \subset \mathfrak{h}_{n c},} \\
\mathfrak{u}_{1}^{\prime} \equiv\left[\mathfrak{u}^{\prime}, \mathfrak{u}^{\prime}\right]=\mathfrak{l}_{1}^{\prime}+\left\{\left(\mathfrak{m}_{1}^{\prime \prime}+\mathfrak{g}_{\mathrm{c}}\right) \cap[\mathfrak{h}, \mathfrak{h}]\right\} \\
\subset \mathfrak{l}_{1}^{\prime}+\left\{\left(\mathfrak{m}_{1}^{\prime \prime}+\mathfrak{g}_{\mathrm{c}}\right) \cap \pi_{1}[\mathfrak{h}, \mathfrak{h}]\right\},
\end{gathered}
$$

where $\pi_{1}: \mathfrak{g} \rightarrow g_{1}$ is the projection parallel to $g_{2} \cdot \pi_{1}\left(\mathfrak{h}_{2}\right) \cap\left(\mathfrak{m}_{1}^{\prime \prime}+g_{c}\right)$ is a solvable ideal in the semisimple algebra $\mathfrak{m}_{1}^{\prime \prime}+\mathfrak{g}_{c}$ and hence is trivial. Therefore, $\left(\mathfrak{m}_{1}^{\prime \prime}+\mathfrak{g}_{c}\right)$ $\cap \pi_{1}[\mathfrak{h}, \mathfrak{h}]=\left(\mathfrak{m}_{1}^{\prime \prime}+\mathfrak{g}_{c}\right) \cap \mathfrak{h}_{1}=\mathfrak{h}_{c}$ by Theorem 3.1. Hence

$$
\left[\mathfrak{u}^{\prime}, \mathfrak{u}^{\prime}\right] \subset \mathfrak{h}_{1} \text {. }
$$

Since $\mathfrak{h}_{c}+(\mathfrak{l} \cap \mathfrak{h}) \subset \mathfrak{u}^{\prime},(3.3)$ and (3.5) imply $\mathfrak{h}_{\mathfrak{l}}+(\mathfrak{h} \cap \mathfrak{l})$ is a subalgebra of $\mathfrak{h}$. Moreover, by (3.2),

$$
\mathfrak{h}_{1}+(\mathfrak{l} \cap \mathfrak{h})=\mathfrak{h}_{n c} \oplus\left\{\left(\mathfrak{l} \cap \mathfrak{h}+\mathfrak{h}_{1}\right) \cap\left(\mathfrak{m}^{\prime \prime}+\mathfrak{g}_{\mathfrak{c}}+\mathfrak{t}\right)\right\} .
$$

The second summand is compact, hence reductive, so $\mathfrak{h}_{1}+(\mathfrak{l} \cap \mathfrak{h})$ is reductive.

3.4 REMARK. In the proof of Theorem 3.3 , we produced a compactly embedded subalgebra $\mathfrak{u}^{\prime}$ of $\mathfrak{h}$ satisfying $\mathfrak{l} \cap \mathfrak{h} \subset \mathfrak{u}^{\prime}=\mathfrak{l}^{\prime} \oplus \mathfrak{h}_{\mathfrak{c}} \oplus \mathfrak{t}^{\prime}$, where $\mathfrak{f}^{\prime}$ is a maximal compactly embedded subalgebra of $\mathfrak{h}_{n c}$ and $t^{\prime} \equiv \mathfrak{u}^{\prime} \cap \mathfrak{h}_{2}$ is abelian. Thus the conclusion of Lemma 2.2 remains true when we replace the hypothesis that $L$ be compact by the hypothesis that $G$ be a transitive effective group of isometries of a Riemannian manifold with isotropy subgroup $L$.

4. Transitive subgroups with equal noncompact semisimple parts. The problem of describing the embedding of $H$ in $G$ is complicated by the fact that the solvable radical $H_{2}$ can project nontrivially onto each of $G_{\mathrm{nc}}, G_{\mathrm{c}}$ and $G_{2}$. To alleviate this difficulty, we first shrink $G$ to the largest subgroup $F$ satisfying $F_{\mathrm{nc}}=H_{\mathrm{nc}}$. Proposition 4.2 will show that $F$ is canonically embedded in $G$. We will then investigate the embedding of $H$ in $F$.

4.1 Definition. Subgroups $H$ and $\tilde{H}$ of $G$ with $H \subset \tilde{H}$ will be said to have the same noncompact semisimple part if some choice of semisimple Levi factors $H_{1}$ and $\tilde{H}_{1}$ with $H_{1} \subset \tilde{H}_{1}$ satisfies $H_{\mathrm{nc}}=\tilde{H}_{\mathrm{nc}}$ (see Notation 1.1 ). We will also say that their Lie algebras $\mathfrak{h}$ and $\tilde{\mathfrak{h}}$ have the same noncompact part. Note that the definition is independent of the choice of $H_{1}$ and $\tilde{H}_{1}$. Indeed, if $H_{1}^{\prime}$ and $\tilde{H}_{1}^{\prime}$ are another pair of Levi factors with $H_{1}^{\prime} \subset \tilde{H}_{1}^{\prime}$, then necessarily $H_{\mathrm{nc}}^{\prime} \subset \tilde{H}_{\mathrm{nc}}^{\prime}$. By the conjugacy of Levi factors in any connected Lie group, the dimension of the noncompact parts is independent of the choice of Levi factors and hence $H_{\mathrm{nc}}=\tilde{H}_{\mathrm{nc}}$ iff $H_{\mathrm{nc}}^{\prime}=\tilde{H}_{\mathrm{nc}}^{\prime}$.

4.2 Proposition. Let $G$ be a connected Lie group of the form $G=H L$ with $H$ connected and L compact. We use Notation 1.1. G contains a unique connected subgroup $F$ maximal with respect to the property of having the same noncompact part as 
H. If $H_{1}$ and $G_{1}$ are any Levi factors of $H$ and $G$ with $H_{1} \subset G_{1}$, then there exists an Iwasawa decomposition $G_{n c}=K A N$ such that

$$
F=\left(H_{n c} \cap K\right)(M A N) G_{c} G_{2},
$$

where $M$ is the identity component of the normalizer of $A N$ in $K$. Moreover, F contains the normalizer of $H$ in $G$.

4.3 Remarks. (1) Note that in the notation of Theorem 3.1, $f=h_{\mathrm{nc}}+\mathrm{m}^{\prime \prime}+\mathrm{a}^{\prime \prime}$ $+\mathfrak{n}^{\prime \prime}+\mathfrak{g}_{\mathrm{c}}+\mathfrak{g}_{2}$, vector space direct sum. The assumption in 3.1 that $G_{1}$ be compatible with $L$ is dropped here since each choice of $G_{1}$ is compatible with a suitable conjugate of $L$. Replacing $L$ by a conjugate does not affect the hypothesis of the theorem.

(2) $F$ has Levi decomposition $F_{1} F_{2}$ with $F_{1}=H_{\mathrm{nc}} M_{1}^{\prime \prime} G_{\mathrm{c}}$ and $F_{2}=\left(M_{2}^{\prime \prime} A N\right) G_{2}$, where as usual $M_{1}^{\prime \prime}$ and $M_{2}^{\prime \prime}$ denote the semisimple part and center of the reductive group $M$.

Proof of Proposition 4.2. In the notation of 3.1 let $\pi_{\mathrm{nc}}: \mathfrak{g} \rightarrow \mathfrak{g}_{\mathrm{nc}}$ be the projection parallel to $\mathfrak{g}_{\mathrm{c}}+\mathfrak{g}_{2}$. We claim that the normalizer $\mathfrak{b}$ of $\pi_{\mathrm{nc}}(\mathfrak{h})$ in $\mathfrak{g}_{\mathrm{nc}}$ is contained in $\mathfrak{h}_{n c}+\mathfrak{m}^{\prime \prime}+\mathfrak{a}^{\prime \prime}+\mathfrak{n}^{\prime \prime}$. As in the proof of Theorem 3.1, any subalgebra of $\mathfrak{g}_{\mathrm{nc}}$ containing $\pi_{\mathrm{nc}}(\mathfrak{h})$, in particular $\mathfrak{b}$, is the Lie algebra of a transitive group of isometries of the symmetric space $G_{\mathrm{nc}} / K$ with left-invariant metric. Suppose $\mathfrak{b} \not \subset \mathfrak{h}_{\mathrm{nc}}$ $+m^{\prime \prime}+a^{\prime \prime}+n^{\prime \prime}$. Then by (3.1) and the statements following it, it follows that $\mathfrak{b}_{\mathrm{nc}} \neq \mathfrak{h}_{\mathrm{nc}}$. Hence $\mathfrak{b}_{\mathrm{nc}}$ properly contains $\mathfrak{h}_{\mathrm{nc}}$.

Choose an Iwasawa decomposition $\mathfrak{b}_{\mathrm{nc}}=\tilde{\mathfrak{f}}+\tilde{\mathfrak{a}}+\tilde{\mathfrak{n}}$ such that $\mathfrak{f}^{\prime} \subset \tilde{\mathfrak{f}}, a^{\prime} \subset \tilde{\mathfrak{a}}$, $\mathfrak{n}^{\prime} \subset \tilde{\mathfrak{n}}$ where $\mathfrak{f}^{\prime}+\mathfrak{a}^{\prime}+\mathfrak{n}^{\prime}$ is the Iwasawa decomposition of $\mathfrak{h}_{n}$ given in 3.1. Necessarily $\operatorname{dim} \tilde{n}>\operatorname{dim} n^{\prime}$. Now the nilradical $n^{\prime \prime}$ of $\pi_{\text {nc }}(\mathfrak{h})$ is a nilpotent ideal in the normalizer $\mathfrak{b}$. Since $\mathfrak{b}_{n c}$ contains no nilpotent ideals, $\mathfrak{b}_{n c} \cap \mathfrak{n}^{\prime \prime}=\{0\}$. Thus $\tilde{\mathfrak{n}}+\mathfrak{n}^{\prime \prime}$ is a nilpotent subalgebra of $\mathfrak{b}$ properly containing $n^{\prime}+\mathfrak{n}^{\prime \prime}=\mathfrak{n}$, contradicting the fact that $\mathfrak{n}$ is a maximal nilpotent subalgebra of $\mathfrak{g}_{n c}$. Thus $\mathfrak{b} \subset \mathfrak{h}_{n c}+\mathfrak{m}^{\prime \prime}+$ $a^{\prime \prime}+\mathfrak{n}^{\prime \prime}$ as claimed.

It follows from the claim and Remarks 4.3 that the subalgebra $f$ of $g$ defined by (4.1) contains the normalizer of $\mathfrak{h}$. $\mathfrak{f}$ has Levi factor $\mathfrak{f}_{1}=\mathfrak{h}_{n c}+\mathfrak{m}_{1}^{\prime \prime}+\mathfrak{g}_{\mathrm{c}}$, where $m_{1}^{\prime \prime}=\left[m_{1}, m_{1}\right]$ and $\mathfrak{f}_{n c}=\mathfrak{h}_{n c}$. Moreover, any subalgebra $\tilde{\mathfrak{f}}$ of $\mathfrak{g}$ properly containing $\mathfrak{f}$ satisfies $\pi_{n c}(\mathfrak{h})+\mathfrak{m}^{\prime \prime}+\mathfrak{a}^{\prime \prime}+\mathfrak{n}^{\prime \prime} \subset \tilde{f} \cap \mathfrak{g}_{n c}$. As in the preceding paragraph, it follows that $\tilde{f}$ contains a semisimple subalgebra of noncompact type properly containing $\mathfrak{h}_{n c}$. Thus the analytic subgroup $F$ of $G$ with Lie algebra $\mathfrak{f}$ satisfies the maximality condition of Proposition 4.2.

We are left to prove uniqueness of $F$. Let $F^{\prime}$ be another connected subgroup satisfying the maximality condition. Choose Levi factors $F_{1}^{\prime}$ of $F^{\prime}$ and $G_{1}^{\prime}$ of $G$ with $F_{1}^{\prime} \subset G_{1}^{\prime}$. $\mathfrak{g}_{c}^{\prime}+\mathfrak{g}_{2}$ is a $\mathfrak{g}$-ideal, so $\mathfrak{f}^{\prime \prime} \equiv \mathfrak{f}^{\prime}+\mathfrak{g}_{\mathrm{c}}^{\prime}+\mathfrak{g}_{2}$ is a subalgebra of $\mathrm{g}$. Since $f_{1}^{\prime} \subset g_{1}^{\prime}$ it is easily checked that $f^{\prime \prime}$ has Levi factor $f_{1}^{\prime \prime}=\left(f_{1}^{\prime} \cap g_{n c}^{\prime}\right)+g_{c}^{\prime}$ and $f_{n c}^{\prime \prime}=f_{n c}^{\prime}$. By the maximality of $F^{\prime}, f^{\prime}=f^{\prime \prime}$, i.e., $g_{c}^{\prime}+g_{2} \subset f^{\prime}$. Now there exists $X \in g_{2}$ such that $g_{1}=e^{\text {ad } X}\left(g_{1}^{\prime}\right)$, where $g_{1}$ is the Levi factor in the definition of $f$. $\mathfrak{g}_{\mathrm{c}}=e^{\text {ad } X}\left(\mathfrak{g}_{\mathrm{c}}^{\prime}\right) \subset \mathfrak{g}_{\mathrm{c}}^{\prime}+\mathfrak{g}_{2}$ and hence $\mathfrak{g}_{\mathrm{c}}+\mathfrak{g}_{2}=\mathfrak{g}_{\mathrm{c}}^{\prime}+\mathfrak{g}_{2}$ by a dimensionality check. Therefore, $\mathfrak{g}_{c}+\mathfrak{g}_{2} \subset \mathfrak{f}^{\prime}$ and $\mathfrak{f}^{\prime}=\left(\mathfrak{f}^{\prime} \cap \mathfrak{g}_{\mathrm{nc}}\right)+\mathfrak{g}_{\mathrm{c}}+\mathfrak{g}_{2} \cdot \mathfrak{h} \subset \mathfrak{f}^{\prime}$, so $\pi_{\mathrm{nc}}(\mathfrak{h}) \subset \mathfrak{f}^{\prime} \cap \mathfrak{g}_{\mathrm{nc}}$. As in the first part of the proof, it follows that $\mathfrak{f}^{\prime} \cap \mathfrak{g}_{\mathrm{nc}} \subset \mathfrak{h}_{\mathrm{nc}}+\mathfrak{m}^{\prime \prime}+\mathfrak{a}^{\prime \prime}+\mathfrak{n}^{\prime \prime}$ and hence $\mathfrak{f}^{\prime} \subset \mathfrak{f}$. By maximality, $\mathfrak{f}^{\prime}=\mathfrak{f}$. 
Much of the analysis of the embedding of $H$ in $F$ does not require the maximality condition on $F$ but only that $F_{\text {nc }}=H_{\text {nc }}$ and $H \subset F$. In the results below we will replace $F$ by any subgroup with the latter two properties. The greater generality will be needed in part II.

4.4 LemMa. Let $G$ be a connected Lie group of the form $G=H L$ with $H$ connected and $L$ compact and let $\tilde{H}$ be any subgroup of $G$ which has the same noncompact semisimple part as $H$. Choose Levi factors $\mathfrak{h}_{1} \subset \tilde{\mathfrak{h}}_{1}$ of $\mathfrak{h}$ and $\tilde{\mathfrak{h}}$. Then

(i) $\mathfrak{h}_{c} \subset \tilde{\mathfrak{h}}_{c}, \mathfrak{h}_{2} \subset \tilde{\mathfrak{h}}_{c}+\tilde{\mathfrak{h}}_{2}$ and $\left[\mathfrak{h}, \mathfrak{h}_{2}\right] \subset \tilde{\mathfrak{h}}_{2}$.

(ii) If $\tilde{\mathfrak{h}}_{1}$ is compatible with $\tilde{\mathfrak{l}} \equiv \tilde{\mathfrak{h}} \cap \mathfrak{l}$, then $\tilde{\mathfrak{h}}_{1}=\mathfrak{h}_{1}+\tilde{\mathfrak{l}}_{1}$, where $\tilde{\mathfrak{l}}_{1}=[\tilde{\mathfrak{l}}, \tilde{\mathfrak{l}}]$.

Proof. (i) $\mathfrak{h}_{\mathrm{c}} \subset \mathfrak{h}_{1}$ and $\left[\mathfrak{h}_{c}, \tilde{\mathfrak{h}}_{n c}\right]=\left[\mathfrak{h}_{c}, \mathfrak{h}_{n c}\right]=\{0\}$, so $\mathfrak{h}_{c} \subset \tilde{\mathfrak{h}}_{c}$. Let $\tilde{\pi}_{n c}: \tilde{\mathfrak{h}}_{\rightarrow} \rightarrow \tilde{\mathfrak{h}}_{\mathrm{nc}}$ and $\tilde{\pi}_{c}: \tilde{\mathfrak{h}} \rightarrow \tilde{\mathfrak{h}}_{c}$ be the homomorphic projections relative to $\tilde{\mathfrak{h}}=\tilde{\mathfrak{h}}_{\mathrm{nc}}+\tilde{\mathfrak{h}}_{\mathrm{c}}+\tilde{\mathfrak{h}}_{2}$. $\tilde{\mathfrak{h}}_{\mathrm{nc}}=\mathfrak{h}_{\mathrm{nc}}=\tilde{\pi}_{\mathrm{nc}}(\mathfrak{h}) . \tilde{\pi}_{\mathrm{nc}}\left(\mathfrak{h}_{2}\right)$ is a solvable ideal in the semisimple algebra $\tilde{\pi}_{\mathrm{nc}}(\mathfrak{h})$, hence is trivial and $\tilde{\mathfrak{h}}_{2} \subset \tilde{\mathfrak{h}}_{\mathrm{c}}+\tilde{\mathfrak{h}}_{2} \cdot \tilde{\pi}_{\mathrm{c}}(\mathfrak{h})$ is a compact algebra containing $\tilde{\pi}_{\mathrm{c}}\left(\mathfrak{h}_{2}\right)$ as a solvable ideal, so $\tilde{\pi}_{\mathrm{c}}\left(\mathfrak{h}_{2}\right)$ is central in $\tilde{\pi}_{\mathrm{c}}(\mathfrak{h})$. In particular, $\tilde{\pi}_{\mathrm{c}}\left[\mathfrak{h}, \mathfrak{h}_{2}\right]=\left[\tilde{\pi}_{\mathrm{c}}(\mathfrak{h}), \tilde{\pi}_{\mathrm{c}}\left(\mathfrak{h}_{2}\right)\right]$ $=\{0\}$ and $\left[\mathfrak{h}, \mathfrak{h}_{2}\right] \subset \tilde{\mathfrak{h}}_{2}$.

(ii) $\tilde{\mathfrak{h}}_{\mathrm{nc}} \subset \mathfrak{h}$ and $\tilde{\mathfrak{h}}_{\mathrm{c}}=\tilde{\pi}_{\mathrm{c}}(\mathfrak{h})+\tilde{\pi}_{\mathrm{c}}(\tilde{\mathfrak{l}})$.

Theorem 1.1 of Oniščik [On] states that if $\mathfrak{a}, \mathfrak{b}$ and $\mathfrak{c}$ are compact Lie algebras satisfying $\mathfrak{a}=\mathfrak{b}+\mathfrak{c}$, then $[\mathfrak{a}, \mathfrak{a}]=[\mathfrak{b}, \mathfrak{b}]+[\mathfrak{c}, \mathfrak{c}]$. Hence

$$
\tilde{\mathfrak{h}}_{\mathrm{c}}\left(=\left[\tilde{\mathfrak{h}}_{\mathrm{c}}, \tilde{\mathfrak{h}}_{\mathrm{c}}\right]\right)=\left[\tilde{\pi}_{\mathrm{c}}(\mathfrak{h}), \tilde{\pi}_{\mathrm{c}}(\mathfrak{h})\right]+\left[\tilde{\pi}_{\mathrm{c}}(\tilde{\mathfrak{l}}), \tilde{\pi}_{\mathrm{c}}(\tilde{\mathfrak{l}})\right] \text {. }
$$

Since, by (i), $\left[\tilde{\pi}_{\mathrm{c}}(\mathfrak{h}), \tilde{\pi}_{\mathrm{c}}\left(\mathfrak{h}_{2}\right)\right]=\{0\}$ and $\mathfrak{h}_{\mathrm{c}} \subset \tilde{\mathfrak{h}}_{\mathrm{c}}$,

$$
\tilde{\mathfrak{h}}_{\mathrm{c}}=\mathfrak{h}_{\mathrm{c}}+\tilde{\pi}_{\mathrm{c}}\left(\tilde{\mathfrak{l}}_{1}\right) \subset \mathfrak{h}_{\mathrm{c}}+\tilde{\mathfrak{l}}_{1}+\mathfrak{h}_{\mathrm{nc}}=\mathfrak{h}_{1}+\tilde{\mathfrak{l}}_{1} .
$$

Thus $\tilde{\mathfrak{h}}_{1} \subset \mathfrak{h}_{1}+\tilde{\mathfrak{l}}_{1}$. But $\mathfrak{h}_{1}+\tilde{\mathfrak{l}}_{1} \subset \tilde{\mathfrak{h}}_{1}$ by the compatibility condition.

4.5 LEMMA. With the hypotheses of Lemma 4.4, assume that $\tilde{H}_{1}$ is compatible with $\tilde{L}=L \cap \tilde{H}$. Then $\tilde{H}_{1}=H_{1} \tilde{L}_{1}$ and $H_{1}$ is compatible with $H \cap L$.

Proof. We use the notation of the proof of 4.4. Let be a maximal compact subalgebra of $\mathfrak{h}_{n c}$ containing $\tilde{\pi}_{n c}(\tilde{\mathfrak{l}})$. $\tilde{\mathfrak{l}}_{1} \subset \mathfrak{l}+\tilde{\mathfrak{h}}_{c}$ and $\mathfrak{l}+\tilde{\mathfrak{h}}_{c}=\mathfrak{f}+\mathfrak{h}_{c}+\tilde{\mathfrak{l}}_{1}$ by 4.4. Apply Theorem 1.2 of Oniščik [On] (if $B$ and $C$ are subgroups of a Lie group $A$ such that $\mathfrak{a}=\mathfrak{b}+\mathfrak{c}$ and $\mathfrak{a}$ is compact, then $A=B C$ ) to conclude that $K \tilde{H}_{\mathrm{c}}=K H_{\mathrm{c}} \tilde{L}_{1}$. Therefore, $\tilde{H}_{1}=H_{\mathrm{nc}} \tilde{H}_{\mathrm{c}}=\left(H_{\mathrm{nc}} K\right) H_{\mathrm{c}} \tilde{L}_{1}=H_{1} \tilde{L}_{1}$ since $K \subset H_{\mathrm{nc}} \cdot \tilde{\mathfrak{h}}_{1}+\tilde{\mathfrak{l}}=\mathfrak{h}_{1}+\tilde{\mathfrak{l}}$, so $\mathfrak{u} \equiv \mathfrak{h}_{1}+(\mathfrak{l} \cap \mathfrak{h})\left(=\left(\tilde{\mathfrak{h}}_{1}+\tilde{\mathfrak{l}}\right) \cap \mathfrak{h}\right)$ is a subalgebra of $\mathfrak{h}$. Let $\tilde{\mathfrak{l}}=\left(\tilde{\mathfrak{h}}_{1}+\tilde{\mathfrak{l}}\right) \cap \tilde{\mathfrak{h}}_{2} . \mathfrak{f}$ is central in $\tilde{\mathfrak{h}}_{1}+\tilde{\mathfrak{l}}$ and $\mathfrak{u}=\mathfrak{h}_{n c}+\mathfrak{u} \cap\left(\tilde{\mathfrak{h}}_{c}+\tilde{\mathfrak{t}}\right) \cdot \mathfrak{u} \cap\left(\tilde{\mathfrak{h}}_{c}+\tilde{\mathfrak{t}}\right)$ is a compact, hence reductive, Lie algebra commuting with $\mathfrak{h}_{n c}$, so $\mathfrak{u}$ is reductive, i.e., $\mathfrak{h}_{1}$ is compatible with $\tilde{\mathfrak{I}} \cap \mathfrak{h}$.

To describe the embedding of $H$ in $F$, we must study the embeddings of $H_{1}(H \cap L)$ in $F_{1}(F \cap L) \equiv H_{1}(F \cap L)$ and of $H_{2}$ in $F$. Since $\mathfrak{h}_{\mathrm{nc}}=\mathfrak{f}_{\mathrm{nc}}$ splits off $\mathfrak{f}_{1}+(\mathfrak{l} \cap \mathfrak{f})$, the first problem reduces to the compact case already studied by Oniščik. To study the embedding of $\mathrm{H}_{2}$, we need to isolate a solvable subgroup $R$ of $H$ such that $H=H_{1}(H \cap L) R$ and the intersection of $H_{1}(H \cap L)$ with $R$ is minimal. We will see in 4.6 that $R$ must contain the subgroup $V$ of $H$ with Lie algebra $\mathfrak{v}=\left\{\mathfrak{h}_{1}+(\mathfrak{h} \cap \mathfrak{l})\right\} \cap\left[\mathfrak{h}, \mathfrak{h}_{2}\right]$. The following example shows that $\mathfrak{v}$ can be nontrivial. 
EXAMPLE. Let $S$ be a solvable Lie group with nontrivial center $Z$, and suppose $\mathfrak{m} \equiv z \cap[\mathfrak{g}, \mathfrak{g}] \neq\{0\}$. Let $D$ be a lattice in the corresponding central subgroup $W$ of $S$. Let $H$ be a connected Lie group of the form $H=H_{1} \times H_{2}$, where $H_{1}$ is a semisimple Lie group containing a torus $T$ and $H_{2} \cong S / D$. Note that $W / D$ is a torus in $H_{2}$. Let $L_{0}$ be a diagonal subgroup of $T \times W / D$ and define a left-invariant Riemannian metric on $M \equiv H / L_{0}$. Let $G$ be the full connected isometry group of $M ; G=H L$ with $L$ compact and $H \cap L=L_{0} . H_{1} L_{0} \subset H_{1} \times W / D$ so $H_{1}$ is compatible with $L_{0}$, and $0 \neq\left(\mathfrak{h}_{1}+\mathfrak{l}\right) \cap\left[\mathfrak{h}, \mathfrak{h}_{2}\right] \subset \mathfrak{w}$.

4.6 TheOREM. Let $H$ and $\tilde{H}$ be connected transitive subgroups of the full isometry group $G$ of a connected homogeneous Riemannian manifold $M$. Assume $H \subset \tilde{H}$ and $H$ and $\tilde{H}$ have the same noncompact semisimple part. Let $L_{p}$ denote the isotropy subgroup of $G$ at $p \in M$. Choose any Levi factors $\mathfrak{h}_{1}$ and $\tilde{\mathfrak{h}}_{1}$ compatible with $\mathfrak{l}_{p} \cap \mathfrak{h}$ and $\mathfrak{l}_{p} \cap \tilde{\mathfrak{h}}$, respectively. (We are not assuming $\left.\mathfrak{h}_{1} \subset \tilde{\mathfrak{h}}_{1}.\right)$ Set $\mathfrak{u}_{p}=\mathfrak{h}_{1}+\left(\mathfrak{l}_{p} \cap \mathfrak{h}\right), \tilde{\mathfrak{u}}_{p}=\tilde{\mathfrak{h}}_{1}+\left(\mathfrak{l}_{p}\right.$ $\cap \tilde{\mathfrak{h}})$ and $\mathfrak{v}=\mathfrak{u}_{p} \cap\left[\mathfrak{h}, \mathfrak{h}_{2}\right]$. Then:

(i) $\mathfrak{v}$ is independent of both the point $p$ in $M$ and of the choice of $\mathfrak{h}_{1}$ compatible with $\mathfrak{h} \cap \mathfrak{l}_{p}$.

(ii) $\tilde{\mathfrak{u}}_{p} \cap\left[\tilde{\mathfrak{h}}, \tilde{\mathfrak{h}}_{2}\right]=\mathfrak{u}_{p} \cap\left[\mathfrak{h}, \mathfrak{h}_{2}\right](=\mathfrak{v})$.

(iii) $\mathfrak{v} \subset\left[\mathfrak{h}_{2}, \mathfrak{h}_{2}\right] \cap z(\mathfrak{h})$, where $z(\mathfrak{h})$ denotes the center of $\mathfrak{h}$.

(iv) Let $\mathfrak{r}$ be any solvable ideal of $\mathfrak{h}$ satisfying $\mathfrak{h}=\mathfrak{u}_{p}+\mathfrak{r},\left[\mathfrak{h}, \mathfrak{h}_{2}\right] \subset \mathfrak{r}$ and $\mathfrak{u}_{p} \cap \mathfrak{r}$ $=\mathfrak{v}$ (such $\mathfrak{r}$ exist) and let $\tilde{\mathfrak{r}}$ be any solvable ideal of $\tilde{\mathfrak{h}}$ satisfying $\tilde{\mathfrak{h}}=\tilde{\mathfrak{H}}_{p}+\tilde{\mathfrak{r}}$, $\left[\tilde{\mathfrak{h}}, \tilde{\mathfrak{h}}_{2}\right] \subset \tilde{\mathfrak{r}}$ and $\tilde{\mathfrak{u}}_{p} \cap \tilde{\mathfrak{r}}=\mathfrak{v}$. Then $\mathfrak{r}$ is a modification of $\tilde{\mathfrak{r}}$ (see 1.4).

(v) For $R$ the connected subgroup of $H$ corresponding to $\mathfrak{r}$ in (iv), $H_{1} R$ acts transitively on $M$. Conversely, if $Q$ is any solvable subgroup of $H_{2}$ normalized by $H_{1}$ such that $H_{1} Q$ acts transitively on $M$, then $\mathfrak{v} \subset$ q. In particular, $\operatorname{dim} R \leqslant \operatorname{dim} Q$.

4.7 Lemma. Suppose a Lie algebra $\mathfrak{b}$ satisfies $\mathfrak{b}=\mathfrak{j}+\mathfrak{g}, \mathrm{i} \cap \mathfrak{g}=\{0\}$, where $\mathfrak{g}$ is a solvable ideal and $\mathrm{j}$ is either a semisimple or a compactly embedded subalgebra. Then

$$
z(\mathfrak{b}) \cap[\mathfrak{d}, \mathfrak{s}] \subset[\mathfrak{s}, \mathfrak{s}],
$$

where $z(\downarrow)$ denotes the center of $\downarrow$.

Proof. Either hypothesis on $i$ implies that the adjoint action of $i$ on $d$ is

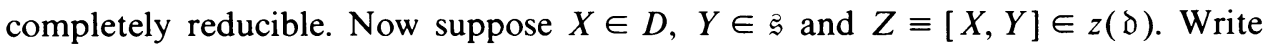

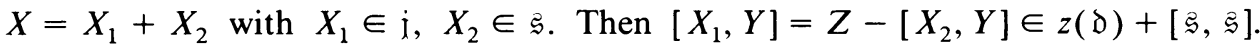
Since each of $\mathfrak{s},[\mathfrak{S}, \mathfrak{g}]$ and $\mathfrak{s} \cap z(\mathfrak{b})$ is $\operatorname{ad}_{\mathfrak{b}}(\mathfrak{i})$-invariant and $[\dot{\mathfrak{i}}, z(\mathfrak{b})]=\{0\}$, the complete reducibility of $\operatorname{ad}_{b} \mathfrak{i}$ implies $[\dot{i}, \mathfrak{g}] \cap(z(\mathfrak{d})+[\mathfrak{I}, \mathfrak{g}]) \subset[\mathfrak{I}, \mathfrak{g}]$ and hence $[X, Y] \in[\mathfrak{I}, \mathfrak{g}]$.

Proof of Theorem 4.6. By Proposition $2.3, \mathfrak{b}$ is compactly embedded in $\mathfrak{h}$, so $\operatorname{ad}_{\mathfrak{h}} \mathfrak{b}$ consists of semisimple operators (skew-symmetric relative to a suitable inner product on $\mathfrak{h})$. But $\mathfrak{v}$ lies in the nilradical of $\mathfrak{h}$, so the operators in $\operatorname{ad}_{\mathfrak{h}} \mathfrak{v}$ are also nilpotent. Thus $\operatorname{ad}_{\mathfrak{h}} \mathfrak{v}=0$ and $\mathfrak{v} \subset z(\mathfrak{h})$, i.e., $\mathfrak{v}=\left(\mathfrak{u}_{p} \cap z(\mathfrak{h})\right) \cap\left[\mathfrak{h}, \mathfrak{h}_{2}\right]$. By Proposition 2.4, $\mathfrak{u}_{p} \cap z(\mathfrak{h})$ and therefore also $\mathfrak{v}$ are independent of the choice of $\mathfrak{h}_{1}$ compatible with $\mathfrak{h} \cap \mathfrak{l}$.

If $\mathfrak{l}_{q}$ is the isotropy subalgebra at another point $q \in M$, then $\mathfrak{l}_{q}=\operatorname{ad}(x) \mathfrak{l}_{p}$ for some $x \in H$, and $\mathfrak{l}_{q} \cap \mathfrak{h}=\operatorname{Ad}(x)\left(\mathfrak{l}_{p} \cap \mathfrak{h}\right)$. $\operatorname{Ad}(x) \mathfrak{h}_{1}$ is compatible with $\mathfrak{l}_{q} \cap \mathfrak{h}$. 
$\mathfrak{u}_{q}=\operatorname{Ad}(x) \mathfrak{u}_{p}=\operatorname{Ad}(x) \mathfrak{h}_{1}+\left(\mathfrak{l}_{q} \cap \mathfrak{h}\right)$. Since $\left[\mathfrak{h}, \mathfrak{h}_{2}\right]$ is an $\mathfrak{h}$-ideal, $\mathfrak{u}_{q} \cap\left[\mathfrak{h}, \mathfrak{h}_{2}\right]=$ $\operatorname{Ad}(x)\left(\mathfrak{u}_{p} \cap\left[\mathfrak{h}, \mathfrak{h}_{2}\right]\right)=\operatorname{Ad}(x) \mathfrak{v}$. But $\operatorname{Ad}(x) \mathfrak{v}=\mathfrak{v}$ since $\mathfrak{v} \subset z(\mathfrak{h})$. Hence $\mathfrak{v}$ is independent of the choice of point $p$.

From now on we will drop the subscript $p$. By (i), we may assume that $\mathfrak{h}_{1} \subset \tilde{\mathfrak{h}}_{1}$. Write $\tilde{\mathfrak{l}}=\mathfrak{l} \cap \tilde{\mathfrak{h}}$. Lemma 4.4 asserts that $\tilde{\mathfrak{h}}_{1}=\mathfrak{h}_{1}+\tilde{\mathfrak{l}}_{1}$. Therefore

$$
\tilde{\mathfrak{u}}=\mathfrak{h}_{1}+\tilde{\mathfrak{l}} \text { and } \mathfrak{u}=\tilde{\mathfrak{u}} \cap \mathfrak{h} .
$$

Set $\tilde{\mathfrak{b}}=\tilde{\mathfrak{u}} \cap\left[\tilde{\mathfrak{h}}, \tilde{\mathfrak{h}}_{2}\right]$. The argument above shows that $\tilde{\mathfrak{b}} \subset z(\tilde{\mathfrak{h}})$. To see that $\mathfrak{v} \subset \tilde{\mathfrak{b}}$, note that $\left[\mathfrak{h}, \mathfrak{h}_{2}\right]$ lies in both $[\tilde{\mathfrak{h}}, \tilde{\mathfrak{h}}]$ and in $\tilde{\mathfrak{h}}_{2}$ by Lemma 4.4 . Hence $\left[\mathfrak{h}, \mathfrak{h}_{2}\right] \subset\left[\tilde{\mathfrak{h}}_{,} \tilde{\mathfrak{h}}_{2}\right]$ and

$$
\mathfrak{v} \subset \tilde{\mathfrak{u}} \cap\left[\tilde{\mathfrak{h}}, \tilde{\mathfrak{h}}_{2}\right]=\tilde{\mathfrak{b}} .
$$

We will prove the opposite inclusion $\tilde{\mathfrak{b}} \subset \mathfrak{v}$ simultaneously with (iii) and (iv). Let $\tilde{\pi}_{\mathrm{c}}: \tilde{\mathfrak{h}} \rightarrow \tilde{\mathfrak{h}}_{\mathrm{c}}$ be the homomorphic projection along $\tilde{\mathfrak{h}}_{\mathrm{nc}}+\tilde{\mathfrak{h}}_{2}$. Fix a vector space complement $\tilde{\mathfrak{f}}$ of $\tilde{\mathfrak{v}}$ in $\tilde{\mathfrak{u}} \cap \tilde{\mathfrak{h}}_{2}$ and let

$$
\tilde{\mathfrak{c}}=\tilde{\pi}_{\mathrm{c}}\left(\mathfrak{h}_{2}\right)+\tilde{\mathfrak{t}} .
$$

By Proposition 2.3 and the fact that $\mathfrak{f}$ commutes with $\tilde{\pi}_{\mathfrak{c}}\left(\mathfrak{h}_{2}\right)$, $\tilde{\mathfrak{c}}$ is compactly embedded in $\tilde{\mathfrak{h}}$. Set

$$
\tilde{\mathfrak{b}}=\tilde{\mathfrak{c}}+\tilde{\mathfrak{h}}_{2}=\tilde{\pi}_{\mathrm{c}}\left(\mathfrak{h}_{2}\right)+\tilde{\mathfrak{h}}_{2} .
$$

Let $\tilde{\mathfrak{r}}$ be any subspace of $\tilde{\mathfrak{h}}_{2}$ satisfying $\left[\tilde{\mathfrak{h}}, \tilde{\mathfrak{h}}_{2}\right] \subset \tilde{\mathfrak{r}}, \tilde{\mathfrak{h}}=\tilde{\mathfrak{u}}+\tilde{\mathfrak{r}}$ and $\tilde{\mathfrak{u}} \cap \tilde{\mathfrak{r}}=\tilde{\mathfrak{b}}$. Then $\tilde{\mathfrak{r}}$ is an $\tilde{\mathfrak{h}}$-ideal and

$$
\tilde{\mathfrak{b}}=\tilde{\mathfrak{r}}+\tilde{\mathfrak{c}} \quad \text { (vector space direct sum). }
$$

Applying Lemma 4.7 with $\mathfrak{d}=\tilde{\mathfrak{h}}, \dot{\mathfrak{i}}=\tilde{\mathfrak{h}}_{1}, \mathfrak{g}=\tilde{\mathfrak{h}}_{2}$, we have $\tilde{\mathfrak{b}} \subset\left[\tilde{\mathfrak{h}}_{2}, \tilde{\mathfrak{h}}_{2}\right]=\left[\tilde{\mathfrak{h}}_{2}, \tilde{\mathfrak{r}}\right]$. Applying 4.7 again with $\mathfrak{d}=\tilde{\mathfrak{b}}, \mathfrak{j}=\tilde{\mathfrak{c}}$ and $\mathfrak{g}=\tilde{\mathfrak{r}}$, we obtain

$$
\tilde{\mathfrak{v}} \subset[\tilde{\mathfrak{r}}, \tilde{\mathfrak{r}}] \text {. }
$$

Now let $\mathfrak{r}$ be any subspace of $\mathfrak{h}_{2}$ satisfying $\left[\mathfrak{h}, \mathfrak{h}_{2}\right] \subset \mathfrak{r}, \mathfrak{u} \cap \mathfrak{r}=\mathfrak{v}$ and $\mathfrak{h}=\mathfrak{u}+\mathfrak{r}$. Since $\mathfrak{h}$ is a transitive subalgebra of $\mathfrak{g},(4.2)$ implies

$$
\tilde{\mathfrak{h}}=\mathfrak{h}+\tilde{\mathfrak{l}}=\tilde{\mathfrak{u}}+\mathfrak{r} \text { and } \tilde{\mathfrak{u}} \cap \mathfrak{r}=\mathfrak{u} \cap \mathfrak{r}=\mathfrak{v} \text {. }
$$

By (4.3), $\tilde{\mathfrak{u}} \cap \mathrm{r} \subset \tilde{\mathfrak{v}}$, so $\mathrm{r} \cap \tilde{\mathfrak{c}}=\{0\}$.

We claim that $\mathfrak{r}+\tilde{\mathfrak{b}}$ is a modification of $\tilde{\mathfrak{r}}$. By (4.4), $\mathfrak{r} \subset \tilde{\mathfrak{b}}$. Thus by (7), $\tilde{\mathfrak{b}}=\mathfrak{r}+(\tilde{\mathfrak{b}} \cap \tilde{\mathfrak{u}})=\mathfrak{r}+\tilde{\mathfrak{b}}+\tilde{\mathfrak{c}}$ with $(\mathfrak{r}+\tilde{\mathfrak{b}}) \cap \tilde{\mathfrak{c}}=\{0\}$. The claim follows from (4.5) since $\tilde{\mathfrak{r}}$ is a $\tilde{\mathfrak{b}}$-ideal.

We next show that $\tilde{\mathfrak{v}} \subset \mathfrak{r}$. By 1.6(ii), there exists a normal modification $\mathfrak{r}^{\prime}$ of $\tilde{\mathfrak{r}}$ such that $\tilde{\mathfrak{b}}+\mathfrak{r}$ is a normal modification of $\mathfrak{r}^{\prime}$. Let $\phi: \tilde{\mathfrak{r}} \rightarrow \tilde{\mathfrak{u}}$ be a modification map associated with the modification $r^{\prime}$ of $\tilde{\mathfrak{r}}$. Apply Lemma 4.7 with $\mathfrak{d}=\phi(\tilde{\mathfrak{r}})+\tilde{\mathfrak{r}}=$ $\phi(\tilde{\mathfrak{r}})+\mathfrak{r}^{\prime}, \dot{\mathfrak{j}}=\phi(\tilde{\mathfrak{r}})$ and $\mathfrak{g}=\mathfrak{r}^{\prime}$ to obtain $\tilde{\mathfrak{b}} \subset\left[\mathfrak{r}^{\prime}, \mathfrak{r}^{\prime}\right]$. Since $\tilde{\mathfrak{v}}+\mathfrak{r}$ is a normal modification of $r^{\prime}$, the same argument shows that

$$
\tilde{\mathfrak{b}} \subset[\mathfrak{r}+\tilde{\mathfrak{b}}, \mathfrak{r}+\tilde{\mathfrak{b}}]=[\mathfrak{r}, \mathfrak{r}] \text {. }
$$

Thus $\mathfrak{r}=\mathfrak{r}+\tilde{\mathfrak{b}}$ is a modification of $\tilde{\mathfrak{r}}$, proving (iv). Moreover (4.7) and (4.8) imply $\tilde{\mathfrak{v}} \subset \mathfrak{v}$, completing the proof of (ii). (iii) is also immediate since $[\mathfrak{r}, \mathfrak{r}] \subset\left[\mathfrak{h}_{2}, \mathfrak{h}_{2}\right]$.

We are left to prove (v). Let $R$ and $U$ denote the connected subgroups of $H$ with Lie algebras $\mathfrak{r}$ and $\mathfrak{u}$. $R$ is normal in $H$, so $H=U R$. Moreover, $\mathfrak{u}=\mathfrak{h}_{1}+(\mathfrak{I} \cap \mathfrak{h})$ 
with $\mathfrak{h}_{1}$ a u-ideal, so $U=H_{1}(L \cap H)$ and $H=\left(H_{1} R\right)(L \cap H)$. Hence $H_{1} R$ is transitive on $M$. Now suppose $H_{1} Q$ is transitive. Apply part (ii) of Theorem 4.6 with $H$ playing the role of $\tilde{H}$ in (ii) and $H_{1} Q$ playing the role of $H$ (thus $Q$ plays the role of $\mathrm{H}_{2}$ ) to conclude that $\mathfrak{v} \subset \mathrm{q}$. Moreover, by (iv), q contains a modification of $r$, so $\operatorname{dim} \mathfrak{q} \geqslant \operatorname{dim} r$.

4.8 Proposition. Let $G$ be the full isometry group of a homogeneous Riemannian manifold $M, H$ a transitive subgroup and $\mathfrak{v}$ the subalgebra defined in Theorem 4.6. Let $\mathfrak{g}_{1}$ be a Levi factor of $\mathfrak{g}$ compatible with the isotropy subalgebra $\mathfrak{l}$ of $\mathfrak{g}$ at some point of $M$. Then

$$
\mathfrak{v} \subset\left(\mathfrak{g}_{1}+\mathfrak{l}\right) \cap\left[\mathfrak{g}, \mathfrak{g}_{2}\right]
$$

Proof. By 4.6(ii), we may assume that $\mathfrak{h}=\mathfrak{f}$, where $\mathfrak{f}$ is the subalgebra of $\mathfrak{g}$ defined in 4.2. We may choose an Iwasawa decomposition $\mathfrak{g}_{\mathrm{nc}}=\mathfrak{f}+\mathfrak{a}+\mathfrak{n}$ such that $\mathfrak{l} \subset \mathfrak{f}+\mathfrak{g}_{\mathrm{c}}+\mathfrak{t}$, where $\mathrm{t}$ is an abelian subalgebra of $\mathfrak{g}_{2}$, commuting with $\mathfrak{g}_{1}$. By $4.2, \mathfrak{h}=\mathfrak{h}_{n c}+\mathfrak{m}^{\prime \prime}+\mathfrak{a}^{\prime \prime}+\mathfrak{n}^{\prime \prime}+\mathfrak{g}_{\mathrm{c}}+\mathfrak{g}_{2}$ and $\mathfrak{h}_{\mathrm{nc}}=\mathfrak{l}^{\prime}+\mathfrak{a}^{\prime}+\mathfrak{n}^{\prime}$ with $\mathfrak{l}^{\prime} \subset \mathfrak{l}, \mathfrak{a}^{\prime} \subset$ $a, \mathfrak{n}^{\prime} \subset \mathfrak{n}$. Hence $\mathfrak{l} \cap \mathfrak{h} \subset \mathfrak{l}^{\prime}+\mathfrak{m}^{\prime \prime}+\mathfrak{g}_{\mathrm{c}}+\mathfrak{t}$ and the Levi factor $\mathfrak{h}_{1}=\mathfrak{h}_{n c}+\mathfrak{m}_{1}^{\prime \prime}+\mathfrak{g}_{c}$ of $\mathfrak{h}$ is compatible with $\mathfrak{l} \cap \mathfrak{h}$. $\mathfrak{v} \subset \mathfrak{h}_{1}+(\mathfrak{l} \cap \mathfrak{h}) \subset \mathfrak{g}_{\mathfrak{l}}+\mathfrak{l}$. Moreover, from the construction of $\mathfrak{h}=\mathfrak{f}$, one sees that the center of $\mathfrak{h}$ lies in $\mathfrak{g}_{2}$, so $\mathfrak{v} \subset\left[\mathfrak{h}, \mathfrak{h}_{2}\right] \cap \mathfrak{g}_{2} \subset$ $[\mathfrak{g}, \mathfrak{g}] \cap \mathfrak{g}_{2}=\left[\mathfrak{g}, \mathfrak{g}_{2}\right]$.

In part II we will isolate a canonical solvable normal subgroup $S$ of $F$ with $F=F_{1}(F \cap L) S, F_{1}(F \cap L) \cap S=V$. By Theorem 4.6, any subgroup $R$ of $H$ satisfying the conditions of 4.6(iv) and (v) is a modification of $S$. Proposition 1.6 says that $S$ can be retrieved from $R$ by at most two suitably chosen normal modifications. We will give a simple algorithm for performing the modifications.

\section{BIBLIOGRAPHY}

[G] C. S. Gordon, Riemannian isometry groups containing transitive reductive subgroups, Math. Ann. 248 (1980), 185-192.

[GW] C. S. Gordon and E. N. Wilson, Isometry groups of Riemannian solvmanifolds, in preparation.

[H] S. Helgason, Differential geometry, Lie groups, and symmetric spaces, Academic Press, New York, 1978.

[J] N. Jacobson, Lie algebras, Interscience, New York, 1962.

[OT] T. Ochiai and T. Takahashi, The group of isometries of a left invariant Riemannian metric on a Lie group, Math. Ann. 223 (1976), 91-96.

[On] A. L. Oniščik, Inclusion relations among transitive compact transformation groups, Amer. Math. Soc. Transl. (2) 50 (1966), 5-58.

[Oz] H. Ozeki, On a transitive transformation group of a compact group manifold, Osaka J. Math. 14 (1977), 519-531.

Department of Mathematics, Washington University, St. Louis, Missouri 63130 